\title{
Cyclooxygenase-2 expression is suppressed by eicosapentaenoic acid and is a prognostic factor in patients with esophageal squamous cell carcinoma after curative surgery
}

\author{
Authors: \\ Koji Mizoguchi, Hideyuki Ishiguro*, Ryo Ogawa, Tatsuya Tanaka, \\ Hiroyuki Sagawa, Tomotaka Okubo, Yosuke Samoto,
}

\section{Affiliation of all authors:}

Gastroenterological Surgery, Nagoya City University Graduate School of Medical Sciences, Nagoya 467-8601, Japan

*Correspondence to:

\section{Hideyuki Ishiguro}

Gastroenterological Surgery, Nagoya City University Graduate School of Medical Sciences

Kawasumi 1, Mizuho-cho, Mizuho-ku, Nagoya 467-8601, Japan

Tel: +81-52-853-8226

Fax: +81-52-842-3906

Email: h-ishi@med.nagoya-cu.ac.jp

\section{Authors e-mails :}

Koji Mizoguchi_E-mail: kozy.m06@gmail.com

Hideyuki Ishiguro* E-mail: $\underline{\text { h-ishi@med.nagoya-cu.ac.jp }}$

Ryo Ogawa E-mail: $\underline{\text { r_smliver@yahoo.co.jp }}$

Tatsuya Tanaka E-mail: tatsuyatanaka8@gmail.com

Hiroyuki Sagawa E-mail: hiro18@med.nagoya-cu.ac.jp

Tomotaka Okubo E-mail: okbtmtk@med.nagoya-cu.ac.jp

Yosuke Samoto E-mail: samosuke.0303@tg.commufa.jp 


\section{ABSTRACT \\ Background/Purpose}

We previously showed that eicosapentaenoic acid (EPA) stimulates apoptosis via caspases in esophageal squamous cell carcinoma (ESCC) cell lines, indicating an anticancer effect. In the present study, we aimed to clarify whether the anticancer and anti-inflammatory actions of EPA were related to cyclooxygenase (COX)-2.

\section{Materials and methods}

We performed immunohistochemical analysis in 92 ESCC specimens to investigate the relationship between COX-2 expression and prognosis. Western blotting in ESCC cell lines was used to determine whether EPA suppressed COX-2 expression.

\section{Results}

Immunohistochemistry showed that COX-2 expression levels were enhanced in ESCC specimens, with a significant prognostic impact on the survival of surgically treated cases $(P$ $=0.0017$, log-rank test). Multivariate analysis showed that the prognostic impact of COX-2 was independent of disease stage $(P=0.02)$. Western blotting revealed that the KYSE180 and TE11 ESCC cell lines expressed COX-2 and that COX-2 expression was greatly suppressed by EPA stimulation.

\section{Conclusions}

The expression of COX-2 was associated with poor survival rates in patients with ESCC. EPA may have anticancer effects via suppression of COX-2.

Key Words: cyclooxygenase-2, eicosapentaenoic acid, esophageal squamous cell carcinoma, immunohistochemistry, prognosis 


\section{Introduction}

In Japan, esophageal squamous cell carcinoma (ESCC) is one of the most lethal malignancies, with a 5-year survival rate of $20-30 \%$ after curative surgery (Isono, Sato, \& Nakayama, 1991; Pisani, Parkin, Bray, \& Ferlay, 1999). During the early stages of esophageal cancer, many patients develop local tumor recurrence or distant metastases soon after surgery.

However in recent years, esophageal cancer treatments have improved using combined modality therapy (Hofstetter et al., 2002; Sio et al., 2016). To receive adequate treatment, it is necessary for patients to maintain good physical health. If the patient's nutritional status is maintained, neoadjuvant chemotherapy can show satisfactory effects (Hamai et al., 2013; Kobayashi et al., 2013). In addition, improvement in perioperative nutritional management contributes to a faster recovery after surgical treatment (Fujita, Daiko, \& Nishimura, 2012).

Recently, considering the need for an optimal nutritional status in patients with esophageal cancer who are receiving medical attention, the importance of nutritional therapies for esophageal cancer has become more apparent. Importantly, patients have been encouraged to take omega-3 polyunsaturated fatty acids (PUFAs), such as eicosapentaenoic acid (EPA) and docosahexaenoic acid (DHA) (Fietkau et al., 2013). In addition, omega-3 PUFAs induce the expression of a family of anti-inflammatory mediators called resolvins, suggesting that omega-3 PUFAs are potentially potent anti-inflammatory agents (Das, 2006).

EPA plays a role in the inhibition of cell proliferation and has therefore attracted much attention in recent years owing to its anticancer effects (Eser, Vanden Heuvel, Araujo, \& Thompson, 2013; Hawcroft et al., 2012; Slagsvold, Pettersen, Follestad, Krokan, \& Schonberg, 2009; Song et al., 2011). In our previous studies, we demonstrated the anticancer and apoptotic effects of EPA in ESCC (Mizoguchi et al., 2014). Therefore, in this study, because EPA is thought to regulate cyclooxygenase- 2 (COX-2) expression, we performed further analyses of the relationship between COX-2 and EPA.

\section{Materials and Methods}

\subsection{Patients and tumor samples}

ESCC specimens were obtained from 92 patients who underwent surgery at Nagoya City University Hospital (Nagoya, Japan) between January 2000 and December 2007. Disease stage was determined after pathologic evaluation of the resected specimens according to the Guidelines for Clinical and Pathological Studies on Esophageal Carcinoma and the TNM classification, sixth edition. The cohort consisted of 73 men and 19 women, with an age at diagnosis ranging from 45 to 79 years (median age, 63 years). 
Thirty-five patients had stage I disease, 22 had stage II disease, 30 had stage III disease, and 5 had stage IV disease.

\subsection{Immunohistochemistry}

ESCC sections were obtained from Nagoya City University Hospital (Nagoya, Japan). For antigen unmasking, deparaffinized sections were boiled in citrate buffer $(10 \mathrm{mM}$ sodium citrate buffer, $\mathrm{pH}$ 6.0) before incubation with primary antibodies. COX-2 protein levels were examined using rabbit monoclonal antibodies (\#12282S; Cell Signaling Technology, Danvers, MA, USA). Antibody staining was performed using the DAKO EnVision system with a peroxidase-labeled polymer (Dako A/S, Glostrup, Denmark).

\subsection{Statistical analysis}

The following biostatistical analyses were performed using R software (Kanda, 2013). The associations between various clinicopathological characteristics and strong COX-2 expression were examined by Fisher's exact test. Kaplan-Meier estimates of overall survival were compared using the log-rank test. Cox regression analysis was used to identify the independent factors with a significant joint effect on survival. The significance level was set at $P<0.01$.

\subsection{Reagents}

5,8,11,14,17-Eicosapentaenoic acid (EPA) was purchased from Sigma Aldrich
(St. Louis, MO, USA) and diluted in ethanol. Antibodies against COX-2 and GAPDH were purchased from Cell Signaling Technology. Polyclonal goat anti-rabbit immunoglobulin/horseradish peroxidase (HRP)-conjugated secondary antibodies was purchased from Dako A/S.

\subsection{Cell culture}

The human esophageal carcinoma cell line TE series was a gift from the Institute of Development, Aging and Cancer, Tohoku University, and the KYSE series was obtained from the Japanese Collection Research Bioresources. Cell lines were cultured in RPMI 1640 medium (Sigma) supplemented with $10 \%$ fetal bovine serum (Gibco Life Technologies, Inc., Grand Island, NY, USA) and antibiotic-antimycotic solution (100×; Sigma). Cell lines were cultured according to the manufacturers' instructions.

\subsection{Cell proliferation}

Cells were seeded in 6-cm dishes at $1.0 \times 10^{5} /$ well and incubated for $24 \mathrm{~h}$. The medium was changed to serum-free medium $24 \mathrm{~h}$ after seeding and incubated for another $24 \mathrm{~h}$. The cells were then exposed to different EPA concentrations (10, 50, and $100 \mu \mathrm{M})$ in serum-free medium for $24 \mathrm{~h}$. After $24 \mathrm{~h}$ of exposure to EPA, Giemsa staining was performed. Cells were fixed with methanol for $5 \mathrm{~min}$. After drying the dishes, Giemsa staining solution (diluted 1:20) was poured onto the dishes, and cells were then incubated 
for $30 \mathrm{~min}$. Cells were washed with tap water and then dried.

\subsection{Western blot analysis}

Equal amounts of protein from total cell lysates solubilized in RIPA buffer were subjected to sodium dodecyl sulfate polyacrylamide gel electrophoresis (SDS-PAGE) and then transferred to nitrocellulose membranes (Bio-Rad, Hercules, CA, USA). The membranes were blocked for $30 \mathrm{~min}$ in phosphate-buffered saline (PBS) containing $5 \%$ milk and were then incubated overnight at $4{ }^{\circ} \mathrm{C}$ with primary antibodies against $\mathrm{COX}-2$ and GAPDH diluted $1: 1000$ in TBS-T $(0.1 \%$ Tween 20) containing $1 \%$ milk. The membranes were then incubated with polyclonal goat anti-rabbit immunoglobulin/HRP-conjugated

Figure 1

(A) COX-2 positive

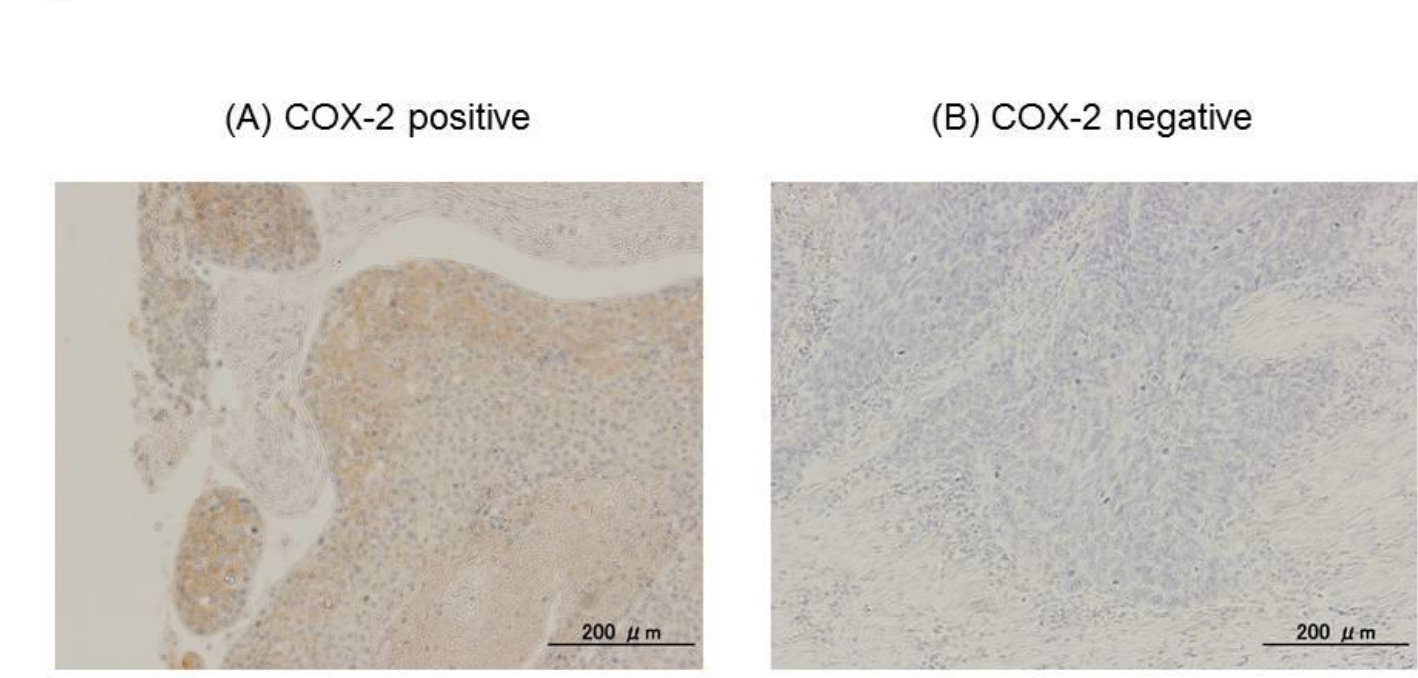

secondary antibodies in TBS-T $(0.1 \%$ Tween 20) containing 1\% milk for $60 \mathrm{~min}$ at room temperature. The protein-antibody complexes on the membranes were visualized using SuperSignal West Pico Chemiluminescent Substrate (Thermo Fisher Scientific, Rockford, IL, USA) and SuperSignal West Femto Chemiluminescent Substrate (Thermo Fisher Scientific).

\section{Results}

\subsection{Relationships between} clinicopathological factors and COX-2 expression

According to immunohistochemical analysis, COX-2 was strongly expressed in $58(63 \%)$ cases and not expressed in 34 $(37 \%)$ cases (Figure 1).

Figure 1. Immunohistochemical staining of COX-2 was positive in 58 cases (63\%) (A) but weak or negative in 34 cases (39\%) (B). 
First, we analyzed the relationships between clinicopathological factors and COX-2 expression in ESCC (Table 1). There were no correlations between the expression of COX-2 and sex, age, histology, tumor depth, lymph node metastasis, lymphatic and venous invasion, or stage.

Table 1.

Relationship between clinicopathological factors and expression of COX-2

\begin{tabular}{|c|c|c|c|c|}
\hline Factor & Group & $\begin{array}{c}\text { COX-2 } \\
\text { expression } \\
\text { Negative }(n=34)\end{array}$ & $\begin{array}{l}\text { COX-2 expression } \\
\text { Positive }(n=58)\end{array}$ & $P$ value \\
\hline \multirow[t]{2}{*}{ Gender } & Female & 4 & 15 & 0.120 \\
\hline & Male & 30 & 43 & \\
\hline \multirow[t]{2}{*}{ Age } & $>65$ & 18 & 19 & 0.078 \\
\hline & $<65$ & 16 & 39 & \\
\hline \multirow[t]{3}{*}{ Histology } & Moderate & 25 & 31 & 0.189 \\
\hline & Poor & 1 & 5 & \\
\hline & Well & 8 & 22 & \\
\hline \multirow[t]{2}{*}{ pT } & $\mathrm{T} 1$ & 18 & 22 & 0.194 \\
\hline & T2-4 & 16 & 36 & \\
\hline \multirow[t]{2}{*}{$\mathrm{pN}$} & NO & 20 & 26 & 0.280 \\
\hline & $\mathrm{N} 1$ & 14 & 32 & \\
\hline \multirow[t]{2}{*}{ Lymphatic invasion } & Negative & 14 & 18 & 0.369 \\
\hline & Positive & 20 & 40 & \\
\hline \multirow[t]{2}{*}{ Venous invasion } & Negative & 17 & 28 & 1 \\
\hline & Positive & 17 & 30 & \\
\hline \multirow[t]{2}{*}{ Stage } & Stage 1 & 17 & 18 & 0.0798 \\
\hline & $\begin{array}{l}\text { Stages 2- } \\
4\end{array}$ & 17 & 40 & \\
\hline
\end{tabular}

Next, we examined whether the expression level of COX-2 was associated with patient survival after surgery. Kaplan-Meier survival curves demonstrated that the probability of surviving several months postoperatively was significantly lower for patients expressing high levels of COX-2 $(P=$ 0.002 by log-rank test; Figure 2). 
Figure 2

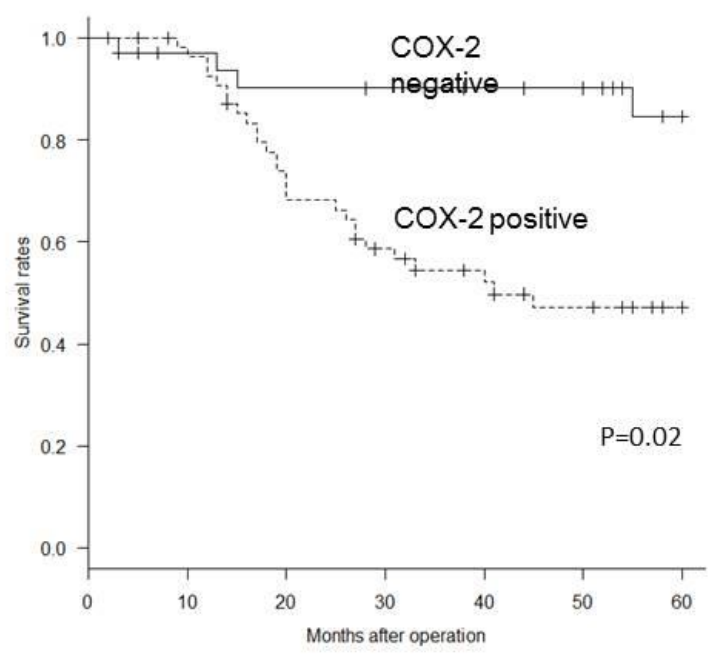

Figure 2. Survival comparisons in the COX-2-negative and -positive groups indicated a significantly poorer prognosis $(P=0.02)$ in the $\mathrm{COX}$-2-positive group.

\subsection{Univariate analysis}

The effects of various clinicopathological factors on prognosis were evaluated using Fisher's exact test (Table 2). According to univariate analysis, tumor status $(P<0.01)$, lymphatic invasion $(P<0.01)$, blood vessel invasion $(P<0.01)$, lymph node status $(P<0.01)$, and COX-2 expression $(P<0.01)$ were predictive factors for poor prognosis. 
COX-2 expression in ESCC

\section{Table 2}

Univariate analysis

\begin{tabular}{|c|c|c|c|}
\hline Parameter & Risk ratio & $95 \% \mathrm{Cl}$ & $P$ value \\
\hline \multicolumn{4}{|l|}{ Age at surgery } \\
\hline$<65$ years & 1 & & \\
\hline$>65$ years & 1.212 & $0.58-2.53$ & 0.61 \\
\hline \multicolumn{4}{|l|}{ Gender } \\
\hline Female & 1 & & \\
\hline Male & 1.22 & $0.50-2.97$ & 0.67 \\
\hline \multicolumn{4}{|l|}{ Tumor status } \\
\hline $\mathrm{T} 1$ & 1 & & \\
\hline T2-4 & 6.605 & $2.31-18.93$ & $<0.01$ \\
\hline \multicolumn{4}{|l|}{ Lymph node status } \\
\hline No & 1 & & \\
\hline N1 & 3.67 & $1.68-7.99$ & $<0.01$ \\
\hline \multicolumn{4}{|l|}{ Lymphatic invasion } \\
\hline Negative & 1 & & \\
\hline Positive & 4.5 & $1.57-12.87$ & $<0.01$ \\
\hline \multicolumn{4}{|l|}{ Blood vessel invasion } \\
\hline Negative & 1 & & \\
\hline Positive & 2.84 & $1.31-6.18$ & $<0.01$ \\
\hline \multicolumn{4}{|l|}{ Stage } \\
\hline Stage 1 & 1 & & \\
\hline Stages 2-4 & 12.45 & $2.96-52.31$ & $<0.01$ \\
\hline \multicolumn{4}{|l|}{ COX-2 expression } \\
\hline Negative & 1 & & \\
\hline Positive & 4.62 & $1.61-13.25$ & $<0.01$ \\
\hline
\end{tabular}

\subsection{Multivariate analysis}

Correlations between prognostic factors and patient overall survival were analyzed using the Cox expression $(P=0.02)$ was identified as a significant and independent prognostic factor in patients who had undergone surgical resection of ESCC (Table 3). 
COX-2 expression in ESCC

\section{Table 3}

Multivariate analysis

\begin{tabular}{llccc} 
Parameter & & Risk ratio & $95 \% \mathrm{Cl}$ & $P$ value \\
\hline Tumor status & & & & \\
& $\mathrm{T} 1$ & 1.13 & $0.25-5.18$ & 0.87 \\
Lymph node status & $\mathrm{T} 2-4$ & & & \\
& $\mathrm{~N} 0$ & 1.46 & $0.59-3.60$ & 0.42 \\
& $\mathrm{~N} 1$ & & & \\
Lymphatic invasion & & & & 0.85 \\
& Negative & 1.14 & $0.29-4.41$ & \\
Vein invasion & Positive & & & 0.42 \\
& Negative & & & \\
Stage & Positive & 1.46 & $0.58-3.69$ & \\
& Stage 1 & & & \\
& Stages & & & 0.121
\end{tabular}

COX-2 expression

\begin{tabular}{llll}
\multicolumn{1}{l}{$\begin{array}{l}\text { Negative } \\
\text { Positive }\end{array}$} & 3.63 & $1.24-10.56$ & 0.02 \\
\hline $\mathrm{Cl}=$ confidence interval & & &
\end{tabular}

3.4 Effects of EPA on COX-2 lines and found that COX-2 was expressed expression

We previously showed that EPA induces apoptosis in ESCC cell lines (14); in this study, we examined the effects of EPA on COX-2 expression. First, we evaluated COX-2 expression in ESCC cell more strongly in KYSE180 cells than in the other ESCC cell lines evaluated in this study (i.e., TE1, TE4, TE5, TE11, KYSE30, KYSE150, KYSE180, KYSE270; Figure 3). 
Figure 3
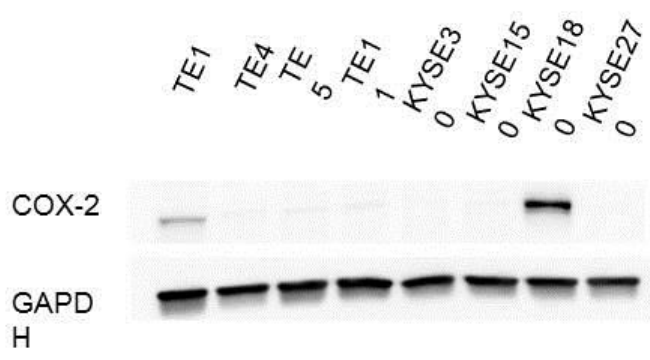

Figure 3. COX-2 protein expression in ESCC cell lines by western blotting. The ESCC cell lines KYSE180 and TE11 strongly expressed COX-2.

Therefore, we used the KYSE180 cell line to evaluate the effects of EPA. KYSE180 cells were exposed to different concentrations of EPA $(0,10$, and 100 $\mu \mathrm{M}$ ) for $24 \mathrm{~h}$ and then subjected to Giemsa staining. Cell growth was suppressed by EPA in a concentration-dependent manner
To confirm the anti-inflammatory effects of EPA, we examined whether the expression of COX-2 was suppressed by EPA. COX-2 protein expression in KYSE180 cells was clearly suppressed by treatment with $10 \mu \mathrm{M}$ EPA after 6 and 12 h (Figure 4B).

(Figure 4A).

Figure 4A

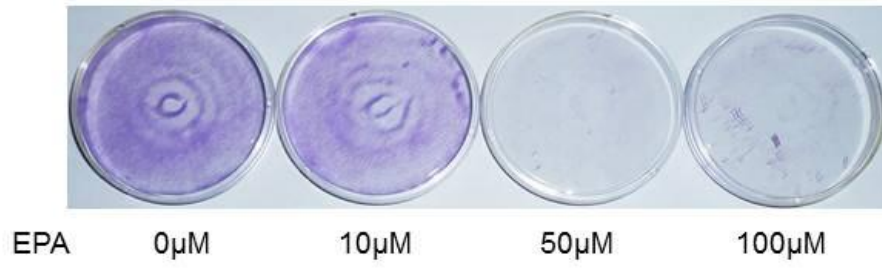

Figure 4B

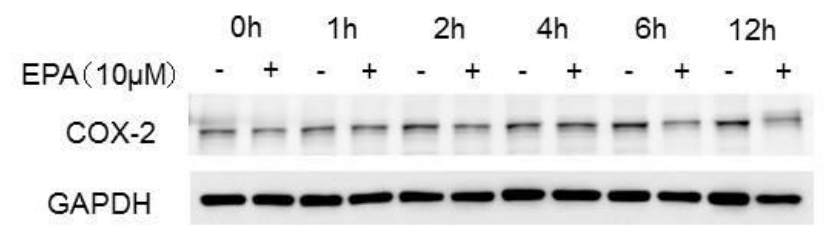

KYSE180

Figure 4. (A) The effects of inhibiting COX-2 expression by EPA. (B) Western blotting showed that COX-2 protein expression in KYSE180 cells was clearly suppressed by treatment with EPA at $10 \mu \mathrm{M}$ after 6 and $12 \mathrm{~h}$. 


\section{Discussion}

COX exists as two isoforms. COX-1 is widely distributed throughout normal body tissues. In contrast, $\mathrm{COX}-2$ is expressed in the brain and kidney and is not usually expressed in other organs. COX-2 expression is induced by inflammatory cytokines and is often overexpressed in tumor tissues. COX-2 leads to production of prostaglandin E2 (PGE2), which is thought to promote carcinogenesis (Nakanishi, Perret, Meuillet, \& Rosenberg, 2015). Inhibition of the COX-2/PGE2 pathway, which can suppress chronic inflammation in cancer tissues, is believed to contribute to inhibition of carcinogenesis. However, to date, COX-2 inhibitors have not been applied clinically in patients with esophageal cancer. Our results suggested that EPA suppressed the proliferation of ESCC cell lines by suppressing COX-2 expression.

We analyzed the expression of COX-2 in 92 specimens excised from patients with ESCC who underwent surgical resection of the esophagus at Nagoya City University Hospital. Patients with strong COX-2 immunohistochemical staining had poorer prognoses than those of patients with weak COX-2 staining.

In our analysis of 92 cases, we found no association between clinicopathological factors and COX-2 expression. According to some reports, COX-2 is correlated with metastasis, tumor depth (Nozoe, Ezaki, Kabashima, Baba, \& Maehara, 2005), and tumor location in esophageal cancer (Sivula et al., 2005). Our results were not completely consistent with other studies (Table 1) but were similar to the results of a study by Takatori et al (2008).

In this study, we detected higher expression levels of COX-2 in $63 \%$ of ESCC specimens. These positive cases were associated with shorter postoperative survival. Notably, among the variables evaluated in the multivariate analysis, COX-2 expression was a significant prognostic factor $(P=0.02)$.

Some previous reports have shown that nonsteroidal anti-inflammatory drugs (NSAIDs) or COX-2 inhibitors reduce the risk of developing esophageal cancer (Oyama et al., 2005; Zhang et al., 2014). Although the functions of COX-2 are still unclear, COX-2 has been shown to stimulate chronic inflammation; therefore, COX-2 inhibitors are thought to suppress inflammation and lead to apoptosis of cancer cells (Zhang et al., 2014).

Our previous study showed that EPA effectively suppresses cancer cell proliferation (Mizoguchi et al., 2014). Based on the effects of COX-2 suppression in ESCC cell lines, we concluded that EPA may exert anticancer effects via suppression of COX-2. Using EPA as an auxiliary of current treatments for esophageal cancer could possibly improve therapeutic effects. 
Although the precise molecular mechanisms of COX-2 upregulation are still unknown, our data indicated that COX-2 was a good candidate molecular prognostic marker and a potential molecular target of therapeutic reagents in patients with this intractable disease.

\section{Acknowledgments}

The authors would like to thank Ms. Seiko Inumaru and Ms. Ryoko Hara for their technical assistance.

The authors declare that they have no competing interest. 


\section{REFERENCES;}

Das, U. N. (2006). Essential fatty acids: biochemistry, physiology and pathology. Biotechnol J, 1(4), 420-439. doi: 10.1002/biot.200600012

Eser, P. O., Vanden Heuvel, J. P., Araujo, J., \& Thompson, J. T. (2013). Marine- and plant-derived omega-3 fatty acids differentially regulate prostate cancer cell proliferation. Mol Clin Oncol, 1(3), 444-452.

doi:

10.3892/mco.2013.76

Fietkau, R., Lewitzki, V., Kuhnt, T., Holscher, T., Hess, C. F., Berger, B., . . . Lubgan, D. (2013). A disease-specific enteral nutrition formula improves nutritional status and functional performance in patients with head and neck and esophageal cancer undergoing chemoradiotherapy: results of a randomized, controlled, multicenter trial. Cancer, 119(18), 3343-3353.

doi:

\section{$10.1002 /$ cncr. 28197}

Fujita, T., Daiko, H., \& Nishimura, M. (2012). Early enteral nutrition reduces the rate of life-threatening complications after thoracic esophagectomy in patients with esophageal cancer. Eur Surg Res, 48(2), 79-84. doi: $10.1159 / 000336574$
Hamai, Y., Hihara, J., Emi, M., Taomoto, J., Aoki, Y., Kishimoto, I., . . . Okada, M. (2013). Treatment outcomes and prognostic factors for thoracic esophageal cancer with clinical evidence of adjacent organ invasion. Anticancer Res, 33(8), 3495-3502.

Hawcroft, G., Volpato, M., Marston, G., Ingram, N., Perry, S. L., Cockbain, A. J., . . Hull, M. A. (2012). The omega-3 polyunsaturated fatty acid eicosapentaenoic acid inhibits mouse MC-26 colorectal cancer cell liver metastasis via inhibition of PGE2-dependent cell motility. $\mathrm{Br} J$ Pharmacol, 166(5), 1724-1737. doi: 10.1111/j.1476-5381.2012.01882. $\mathrm{x}$

Hofstetter, W., Swisher, S. G., Correa, A. M., Hess, K., Putnam, J. B., Jr., Ajani, J. A., . . Roth, J. A. (2002). Treatment outcomes of resected esophageal cancer. Ann Surg, 236(3), 376-384; discussion 384-375. doi: 10.1097/ 01.SLA.0000027925.23604.5C

Isono, K., Sato, H., \& Nakayama, K. (1991). Results of a nationwide study on the three-field lymph node dissection of esophageal cancer. Oncology, 48(5), 411-420. 
Kanda, Y. (2013). Investigation of the freely available easy-to-use software 'EZR' for medical statistics. Bone Marrow Transplant, 48(3), 452-458. doi: 10.1038/bmt.2012.244

Kobayashi, T., Oshima, K., Yokobori, T., Idetsu, A., Hayashi, Y., Hinohara, R. N., . . Kuwano, H. (2013). Perioperative nutriture in esophageal cancer patients undergoing esophagectomy. Hepatogastroenterology, 60(126), 1311-1316. doi: 10.5754/hge11257

Mizoguchi, K., Ishiguro, H., Kimura, M., Takahashi, H., Sakamoto, N., Tanaka, T., \& Takeyama, H. (2014). Induction of apoptosis by eicosapentaenoic acid in esophageal squamous cell carcinoma. Anticancer Res, 34(12), 7145-7149.

Nakanishi, M., Perret, C., Meuillet, E. J., \& Rosenberg, D. W. (2015). Non-cell autonomous effects of targeting inducible PGE2 synthesis during inflammation-associated colon carcinogenesis. Carcinogenesis, 36(4), 478-486. doi: 10.1093/carcin/bgv004

Nozoe, T., Ezaki, T., Kabashima, A., Baba, H., \& Maehara, Y. (2005). Significance of immunohistochemical expression of cyclooxygenase- 2 in squamous cell carcinoma of the esophagus. Am J Surg, 189(1), 110-115. doi: 10.1016/j.amjsurg.2004.03.019

Oyama, K., Fujimura, T., Ninomiya, I., Miyashita, T., Kinami, S., Fushida, S., . . Koichi, M. (2005). A COX-2 inhibitor prevents the esophageal

inflammation-metaplasia-adenocar cinoma sequence in rats. Carcinogenesis, 26(3), 565-570. doi: 10.1093/carcin/bgh340

Pisani, P., Parkin, D. M., Bray, F., \& Ferlay, J. (1999). Estimates of the worldwide mortality from 25 cancers in 1990. Int $J$ Cancer, 83(1), 18-29.

Sio, T. T., Wilson, Z. C., Stauder, M. C., Bhatia, S., Martenson, J. A., Quevedo, J. F., . . . Miller, R. C. (2016). Long-term treatment outcomes for locally advanced esophageal cancer: a single-institution experience. Am J Clin Oncol, 39(5), 448-452. doi: 10.1097/COC.0000000000000089

Sivula, A., Buskens, C. J., van Rees, B. P., Haglund, C., Offerhaus, G. J., van Lanschot, J. J., \& Ristimaki, A. (2005). Prognostic role of cyclooxygenase- 2 in neoadjuvant-treated patients with squamous cell carcinoma of the esophagus. Int J Cancer, 116(6), 903-908. doi: 10.1002/ijc.21134 
Slagsvold, J. E., Pettersen, C. H., Follestad, T., Krokan, H. E., \& Schonberg, S. A. (2009). The antiproliferative effect of EPA in HL60 cells is mediated by alterations in calcium homeostasis. Lipids, 44(2), 103-113. doi: $10.1007 / \mathrm{s} 11745-008-3263-5$

Song, K. S., Jing, K., Kim, J. S., Yun, E. J., Shin, S., Seo, K. S., . . . Lim, K. (2011). Omega-3-polyunsaturated fatty acids suppress pancreatic cancer cell growth in vitro and in vivo via downregulation of Wnt/Beta-catenin signaling. Pancreatology, 11(6), 574-584. doi: $10.1159 / 000334468$

Takatori, H., Natsugoe, S., Okumura, H., Matsumoto, M., Uchikado, Y.,
Setoyama, T., ... Aikou, T. (2008). Cyclooxygenase-2 expression is related to prognosis in patients with esophageal squamous cell carcinoma. Eur J Surg Oncol, 34(4), 397-402. doi: 10.1016/j.ejso.2007.04.011

Zhang, S., Zhang, X. Q., Ding, X. W., Yang, R. K., Huang, S. L., Kastelein, F., . . . Zou, X. P. (2014). Cyclooxygenase inhibitors use is associated with reduced risk of esophageal adenocarcinoma in patients with Barrett's esophagus: a meta-analysis. Br J Cancer, 110(9), 2378-2388. doi: 10.1038/ bjc. 2014.127 